\title{
Dibenzo[hi,st]ovalene as Highly Luminescent Nanographene: Efficient Synthesis via Photochemical Cyclodehydroiodination, Optoelectronic Properties, and Single-Molecule Spectroscopy
}

\author{
Qiang Chen, ${ }^{\#, \dagger \odot ~ S t e f a n ~ T h o m s, ~}{ }^{\#+}$ Sven Stöttinger, ${ }^{\ddagger}$ Dieter Schollmeyer, ${ }^{\S}$ Klaus Müllen, ${ }^{*}, \dagger \oplus$ \\ Akimitsu Narita, ${ }^{* \dagger}+\perp$ a \\ ${ }^{\dagger}$ Max Planck Institute for Polymer Research, Ackermannweg 10, 55128 Mainz, Germany \\ ${ }^{\ddagger}$ Institute of Physical Chemistry, Johannes Gutenberg-University, Duesbergweg 10-14, 55128 Mainz, Germany \\ ${ }^{\S}$ Institute of Organic Chemistry, Johannes Gutenberg-University, Duesbergweg 10-14, 55099 Mainz, Germany \\ ${ }^{\perp}$ Organic and Carbon Nanomaterials Unit, Okinawa Institute of Science and Technology Graduate University, Okinawa 904-0495, \\ Japan
}

\section{Supporting Information}

\begin{abstract}
Dibenzo[hi,st]ovalene (DBOV), as a new nanographene, has demonstrated promising optical properties, such as red emission with a high fluorescence quantum yield of $79 \%$ and stimulated emission, as well as high thermal stability and photostability, which indicated its promise as a light-emitting and optical gain material. However, the previous synthetic routes required at least 12 steps. This obstructed access to different derivatives, e.g., to obtain crystals suitable for X-ray diffraction analysis and to tune the optoelectronic properties. Here, we report an efficient synthetic pathway to DBOV based on a sequential iodination-benzannulation of bi(naphthylphenyl)diyne, followed by photochemical cyclodehydroiodination (PCDHI). This protocol included a fused bischrysene as a key intermediate and furnished scalable amounts of meso-substituted DBOV derivatives with different substituents. DBOV with 2,6-dimethylphenyl groups
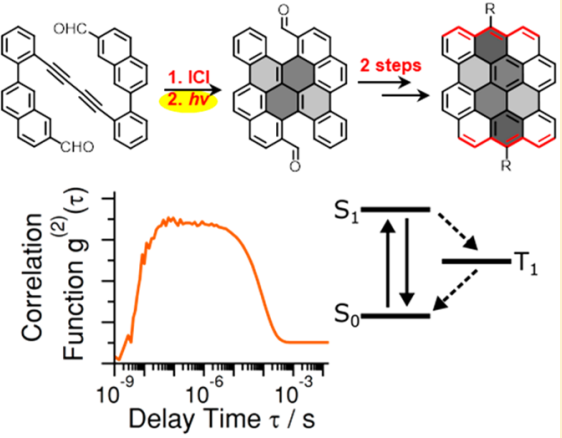
DBOV core. The optoelectronic properties of the DBOV derivatives were investigated by UV-vis absorption and fluorescence spectroscopy, cyclic voltammetry, and density functional theory calculations. Single-molecule spectroscopy at room and low temperatures provided novel insights into the photophysics of DBOV embedded in a polymer film. As a result of weak coupling of the optical transitions to the matrix, single-molecule emission spectra at $4.5 \mathrm{~K}$ showed narrow vibronic lines. The fluorescence autocorrelation function covering 9 orders of magnitude in time displayed high contrast photon antibunching and bunching, from which the fluorescence decay rate and the triplet population and depopulation rates could be retrieved. Remarkably, the intersystem crossing rate into the triplet state decreased by more than an order of magnitude at low temperature, demonstrating that temperature can be a crucial parameter to boost single photon emission of an aromatic hydrocarbon.
\end{abstract}

\section{INTRODUCTION}

Structural confinement of graphene into nanoscale subunits, namely, nanographenes, such as graphene nanoribbons (GNRs) and graphene quantum dots (GQDs), can furnish discrete electronic energy levels with an open bandgap, thus allowing their applications in optoelectronic and photonic devices. ${ }^{1-7}$ Compared with the top-down strategies, such as "cutting" of graphene ${ }^{8-13}$ and "unzipping" of carbon nanotubes, ${ }^{14,15}$ which offer little structural control, sophisticated bottom-up synthesis can produce nanographenes with atomically precise and uniform structures. ${ }^{16-18}$ In recent years, numerous nanographenes have been synthesized in this way, among which nanographene molecules with zigzag edges, i.e., large polycyclic aromatic hydrocarbons (PAHs), such as periacenes $^{19-21}$ and anthenes, ${ }^{22,23}$ have attracted considerable attention for their unique properties, including small energy gaps, unique optical properties, and open-shell biradical character. $^{24,25}$ While nanographene molecules with zigzag edges are often kinetically unstable and subject to immediate oxidation, ${ }^{23,26,27}$ dibenzo[hi,st] ovalene (DBOV) has recently been reported as a highly inert nanographene molecule with a combination of both zigzag and armchair edges. ${ }^{28}$ It exhibited strong red emission with a high absolute fluorescence quantum yield $(\Phi)$ of up to $79 \%$. Moreover, stimulated emission and amplified spontaneous emission (ASE) could be demonstrated, highlighting the potential of DBOV for optoelectronic and photonic applications, such as in light-emitting diodes and lasers. ${ }^{29}$ Nevertheless, the total yield of DBOV was only $2 \%$ after a 12-step synthesis. ${ }^{29}$ This drawback severely hindered

Received: August 2, 2019

Published: October 7, 2019 
further studies into chemical derivatization, crystallization for single-crystal X-ray analysis, and fabrication of optoelectronic devices.

The synthesis of a great majority of nanographene molecules has been performed using the Scholl reaction, namely, intramolecular oxidative cyclodehydrogenation, of predesigned, branched oligoarylene precursors. ${ }^{30-32}$ Whereas numerous nanographene molecules and GNRs could thus be achieved, this method entails significant drawbacks, including the use of excess oxidants, frequently unavoidable peripheral chlorination, rearrangements through aryl migration, low efficiency for electron-deficient systems, and limited scope of functional groups due to the required oxidative conditions. ${ }^{33-37}$ Alternative synthetic methods have been reported, such as acid-promoted cyclization of ethynyl derivatives ${ }^{38,39}$ and metal-catalyzed ${ }^{40,41}$ or photochemical cyclodehydrohalogenation of halogenated precursors. ${ }^{42,43}$ Nevertheless, syntheses of nanographene molecules without Scholl reactions remain scarce. ${ }^{4-46}$

Herein, we report an efficient synthesis of DBOV through a sequence of iodination-benzannulation and photochemical cyclodehydroiodination (PCDHI) without using the Scholl reaction. In the previous synthetic route, to promote the Scholl reaction, we had to convert an electron-withdrawing aldehyde group into an electron-donating acetoxymethyl group (see Scheme 1) and then convert it back. This complication can be avoided in the new method, achieving the synthesis of different DBOV derivatives with alkyl (DBOV-C12), aryl (DBOVDMEP, DBOV-TMOP, DBOV-CF3), and triisopropylsilyl (TIPS)-ethynyl (DBOV-TIPS) groups at the meso-positions in 7 steps, with total yields of $23-41 \%$ (see Scheme 2). DBOV

Scheme 1. Synthesis of Fused Bischrysenyl 6 through a Sequence of ICl-Promoted Iodination-Benzannulation and PCDHI (Red Arrow) vs the Previous Route ${ }^{29}$ via the Scholl Reaction (Blue Arrow) ${ }^{a}$

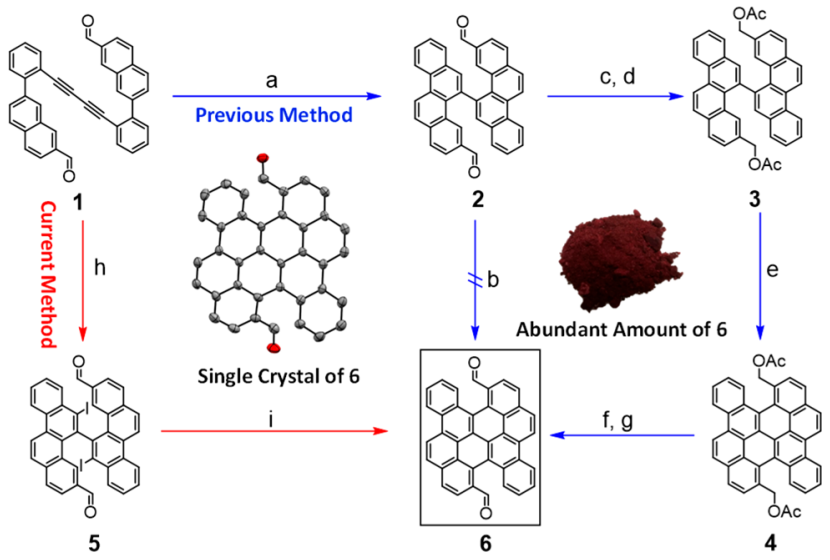

${ }^{a}$ Reagents and conditions: (a) $\mathrm{PtCl}_{2}$, toluene, $80{ }^{\circ} \mathrm{C}, 24 \mathrm{~h}, 45 \%$ yield; (b) $\mathrm{FeCl}_{3}, \mathrm{DCM}$, nitromethane, r.t.; or $\mathrm{DDQ}$ triflic acid, DCM, r.t.; or $h v, \mathrm{I}_{2}$, benzene, $2 \mathrm{~h}$; (c) $\mathrm{NaBH}_{4}$, THF/methanol = 5:1, r.t., $1 \mathrm{~h}$; (d) acetic anhydride, TEA, 4-DMAP, DCM, r.t., $2 \mathrm{~h}, 87 \%$ yield in two steps; (e) DDQ triflic acid, DCM, r.t., $33 \%$ yield; (f) $\mathrm{K}_{2} \mathrm{CO}_{3}$, THF/ methanol = 1:1, r.t., overnight; (g) PCC, DCM, r.t., $2 \mathrm{~h}, 59 \%$ yield in two steps; (h) ICl, DCM, $-78{ }^{\circ} \mathrm{C}, 2 \mathrm{~h}, 76 \%$ yield; (i) TEA, acetone, $h v, 2$ h, 86\% yield. DCM: dichloromethane; DDQ: 2,3-dichloro-5,6dicyano-1,4-benzoquinone; THF: tetrahydrofuran; TEA: triethylamine; 4-DMAP: 4-dimethylaminopyridine; PCC: pyridinium chlorochromate.
Scheme 2. Synthetic Routes toward DBOV Derivatives ${ }^{a}$

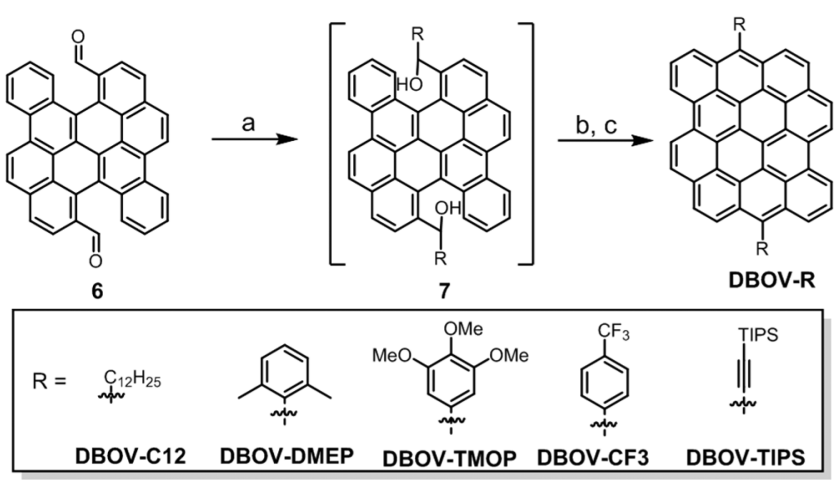

${ }^{a}$ Reagents and conditions: (a) RMgBr or RLi, THF, r.t. 2 h, then $\mathrm{NH}_{4} \mathrm{Cl}, \mathrm{H}_{2} \mathrm{O}$; (b) methanesulfonic acid (MSA) or $\mathrm{BF}_{3} \cdot \mathrm{OEt}_{2}, \mathrm{DCM}$, r.t. $2 \mathrm{~h}$, then $p$-chloranil, r.t., $1 \mathrm{~h}$.

with 2,6-dimethylphenyl units (DBOV-DMEP) provided crystals suitable for single-crystal X-ray analysis. The optical absorption and emission spectra could be fine-tuned by attaching electron-donating or electron-withdrawing aryl substituents, and a redshift of $\sim 40 \mathrm{~nm}$ was achieved by introducing TIPS-ethynyl groups. While the absorption and emission spectra of DBOV have been well characterized, little is known about its intrinsic photophysical kinetics. Therefore, we conducted a comprehensive single-molecule study of immobilized DBOV-DMEP molecules at room and low temperatures. A crucial incentive for single-molecule spectroscopy relates to the fact that spectroscopic and photophysical properties can be retrieved, which are barely accessible from the ensemble average. Fluorescence spectra recorded at $4.5 \mathrm{~K}$ displayed sharp vibronic features dominated by a strong purely electronic zero-phonon line. The fluorescence intensity autocorrelation function covering 9 orders of magnitude in time showed high-contrast photon antibunching at short times and photon bunching due to shelving in the triplet state at long times. Quantitative analysis of the correlation function of single DBOV-DMEP molecules provided access to the lifetimes of the singlet $\left(\mathrm{S}_{1}\right)$ and triplet $\left(\mathrm{T}_{1}\right)$ states as well as the intersystem crossing rate from $S_{1}$ to $T_{1}$. Remarkably, by lowering the temperature, the intersystem crossing (ISC) rate decreased by a factor of $\sim 10$, while the triplet lifetime remained almost constant.

\section{RESULTS AND DISCUSSION}

Photochemical Cyclodehydroiodination (PCDHI). Bi(naphthylphenyl)diyne $\mathbf{1}$ was prepared by $\mathrm{CuCl}$-catalyzed Glaser self-coupling of (2-ethynylphenyl)naphthalene in air. ${ }^{29}$ Afterward, iodination-benzannulation of 1 with $\mathrm{ICl}$ at $-78{ }^{\circ} \mathrm{C}$ provided iodinated bichrysenyl 5 in $76 \%$ yield (Scheme 1 ). Transition-metal-catalyzed cyclization of 5 to fused product 6 was initially attempted by heating with $\mathrm{Pd}\left(\mathrm{PPh}_{3}\right)_{2} \mathrm{Cl}_{2} \cdot \mathrm{CH}_{2} \mathrm{Cl}_{2}$ in the presence of $\mathrm{NaOAc}$ at $140{ }^{\circ} \mathrm{C}$ for $12 \mathrm{~h},{ }^{40}$ which gave only deiodinated bichrysenyl $2 . \operatorname{Pd}\left(\mathrm{PPh}_{3}\right)_{4}$ was next used as a catalyst and gave the same result. Further attempts to planarize 2 through the Scholl reaction (with $\mathrm{FeCl}_{3}$ or DDQ/triflic acid) or photocyclodehydrogenation $\left(\mathrm{I}_{2}, h v\right.$, benzene) also failed, most likely due to the electron-withdrawing nature of the aldehyde groups, consistent with our previous results. ${ }^{28}$ Interestingly, we noticed that the color of diiodobichrysenyl precursor $\mathbf{5}$ on the thin-layer chromatography (TLC) plate 
gradually changed to red when the material was exposed to a hand-held UV lamp, indicating the formation of a large $\pi$ conjugated system. This observation prompted us to explore the photochemical cyclodehydroiodination $(\mathrm{PCDHI})^{43}$ of 5 for the preparation of 6 .

The photoreaction of $\mathbf{5}$ was initially tried with a concentration of $6 \times 10^{-4} \mathrm{M}$ in acetone and aqueous $\mathrm{Na}_{2} \mathrm{CO}_{3}$ solution $(0.1 \mathrm{M}, 1.0$ equiv per PCDHI reaction), which had previously been used as standard conditions for photocyclodehydrohalogenation. ${ }^{42}$ After irradiation with 16 UV lamps $(300 \mathrm{~nm}, 14 \mathrm{~W})$ for $2 \mathrm{~h}$, product 6 precipitated from the reaction solution as a red solid in $73 \%$ yield. Toluene and dichloromethane were then tested as solvents, resulting in relatively low isolated yields of $66 \%$ and $60 \%$, respectively (Table 1). Using acetone as the solvent, the effect of the base

Table 1. Effects of Solvents and Base on Photocyclization from 5 to $6^{a}$

\begin{tabular}{cllc} 
entry & solvent & \multicolumn{1}{c}{ base } & yield $^{b}(\%)$ \\
1 & acetone & $0.1 \mathrm{M} \mathrm{Na}_{2} \mathrm{CO}_{3}$ & 73 \\
2 & toluene & $0.1 \mathrm{M} \mathrm{Na}_{2} \mathrm{CO}_{3}$ & 66 \\
3 & DCM & $0.1 \mathrm{M} \mathrm{Na}_{2} \mathrm{CO}_{3}$ & 60 \\
4 & acetone & TEA & 86 \\
5 & acetone & no base & 10
\end{tabular}

${ }^{a}$ Reaction conditions: Reactions were carried out in the indicated solvents with a concentration of $10^{-4} \mathrm{M}$ under irradiation of UV light $(300 \mathrm{~nm}, 224 \mathrm{~W})$ for $2 \mathrm{~h} .{ }^{b}$ Isolated yield.

was next investigated, and triethylamine gave a higher isolated yield of $86 \%$, presumably due to the formation of a homogeneous solution and its well-known electron-donating ability in photocyclization. ${ }^{47}$ The resulting product 6 could be easily collected by filtration. When no base was added, product 6 further underwent acid-promoted intramolecular FriedelCrafts cyclization to give a black insoluble diketone as a byproduct (see Figure S1). A single crystal of $\mathbf{6}$, obtained by slow diffusion of acetonitrile into an $o$-dichlorobenzene solution, illustrates its twisted core geometry (see inset of Scheme 1, Figure S4). ${ }^{48}$ By using the PCDHI reaction, key intermediate 6 could be prepared on the gram scale, thus affording larger amounts of DBOV with different substituents.

Syntheses and Characterization of DBOV Derivatives. For the synthesis of DBOV derivatives with different substituents, precursor 6 was first reacted with the corresponding Grignard or organolithium reagents and quenched with saturated aqueous solution of $\mathrm{NH}_{4} \mathrm{Cl}$ to give diol 7 (Scheme 2). Subsequently, 7 was treated with methanesulfonic acid (MSA) or $\mathrm{BF}_{3} \cdot \mathrm{OEt}_{2}$ and oxidized by $p$-chloranil in situ to provide the desired DBOV derivatives. Alkyl (DBOV-C 12 $_{12}$, functionalized aryl (DBOV-DMEP, DBOV-TMOP, DBOVCF3), and TIPS-ethynyl (DBOV-TIPS) substituents could be introduced. The products were comprehensively characterized by NMR spectroscopy and high-resolution matrix-assisted laser desorption/ionization time-of-flight mass spectrometry (MALDI-TOF MS).

The ${ }^{1} \mathrm{H}$ NMR spectra showed a strong dependence on the solvents, temperature and peripheral substituents. For example, in deuterated tetrahydrofuran and aromatic solvents (toluene$d_{8}$ and $o$-dichlorobenzene- $d_{4}$ ), sharp and well-resolved signals of DBOV-DMEP were observed, which could be assigned with the help of ${ }^{1} \mathrm{H},{ }^{1} \mathrm{H}$-correlation spectroscopy (COSY) and ${ }^{1} \mathrm{H},{ }^{1} \mathrm{H}$-nuclear Overhauser enhancement spectroscopy
(NOESY) (Figure 1a and Figure S23-S24). However, in chloroform- $d$, the signals became broad, indicating pro-
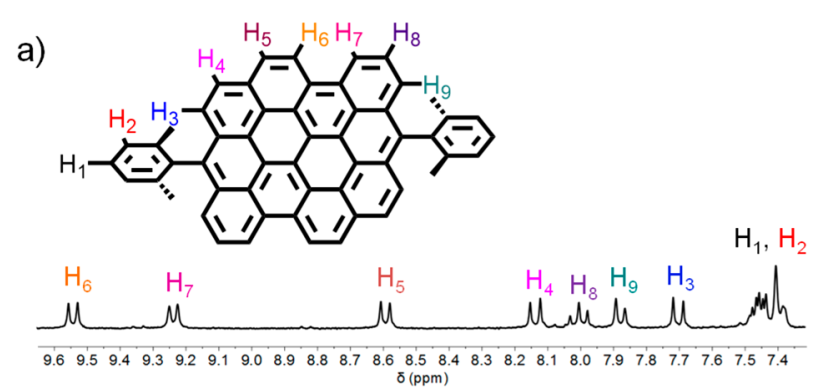

b)
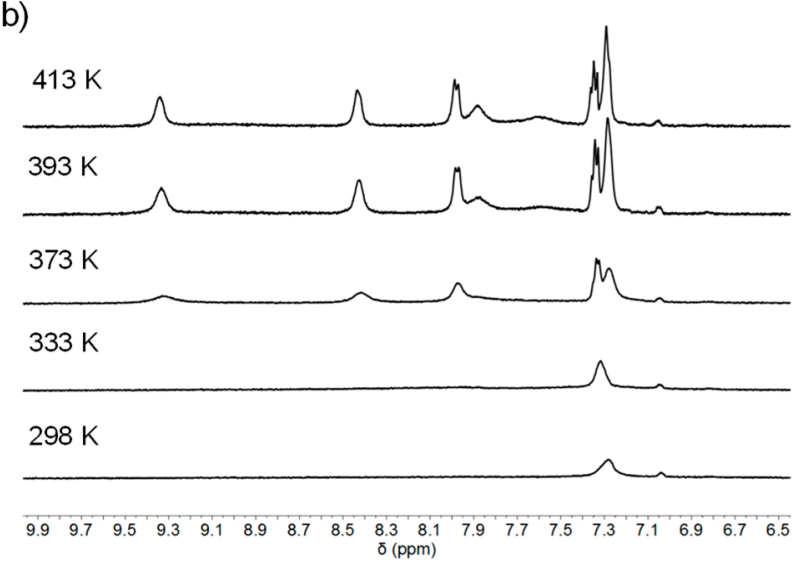

Figure 1. (a) ${ }^{1} \mathrm{H}$ NMR spectrum of DBOV-DMEP in tetrahydrofuran- $d_{8}$ measured at room temperature $(300 \mathrm{MHz})$; (b) variabletemperature ${ }^{1} \mathrm{H}$ NMR spectra $(500 \mathrm{MHz})$ of DBOV-DMEP measured in 1,1,2,2-tetrachloroethane- $d_{2}\left(4.4 \times 10^{-3} \mathrm{M}\right)$.

nounced aggregation of the extended aromatic cores (Figure S2). Variable-temperature ${ }^{1} \mathrm{H}$ NMR spectra of DBOV-DMEP measured in deuterated 1,1,2,2-tetrachloroethane showed one main broad peak at approximately $7.3 \mathrm{ppm}$ at room temperature; upon increasing the temperature, aromatic proton signals of the DBOV core became visible (Figure 1b). However, the peaks were still broad when the temperature reached $413 \mathrm{~K}$ and the resonances moved slightly upfield, as expected from $\pi-\pi$ stacking. Some of the representative zigzag-edged nanographene molecules, such as periacenes and anthenes, ${ }^{22,23}$ are expected to have smaller energy gaps with the potential to generate open-shell ground states with a contribution from thermally accessible triplet biradicals, which often hamper NMR measurements. However, variable-temperature ${ }^{1} \mathrm{H}$ NMR experiments of DBOV-DMEP in $o$ dichlorobenzene- $d_{4}$ showed no obvious signal broadening when increasing the temperature to $423 \mathrm{~K}$ (Figure S3). DFT calculations conducted at the B3LYP/6-31G(d,p) level of theory indicated that the closed-shell form of DBOV is energetically more stable than its open-shell forms, in agreement with the NMR results, accounting for its high stability under ambient conditions (see Table S1).

The structure of DBOV-DMEP was further characterized by $\mathrm{X}$-ray diffraction analysis of crystals obtained by slow diffusion of methanol vapor into a tetrahydrofuran solution of DBOVDMEP and measured at $193 \mathrm{~K}$ (Figure 2a). The DBOVDMEP molecule adopts $C_{2 \mathrm{~h}}$ symmetry with a rigid, almost planar core with the two 2,6-dimethylphenyl groups nearly perpendicular (dihedral angle $\sim 85^{\circ}$ ) as a result of the steric effect of the two methyl groups. The bond lengths are 
a)
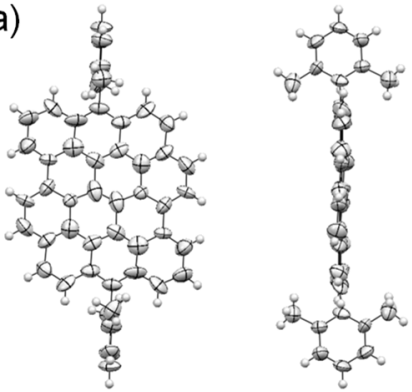

b)

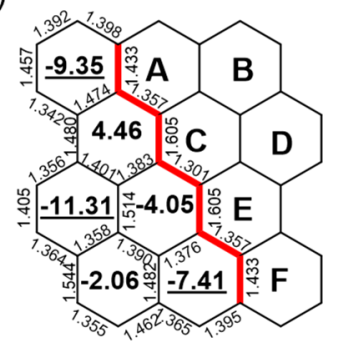

c)

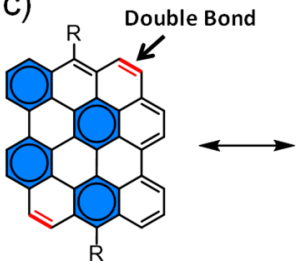

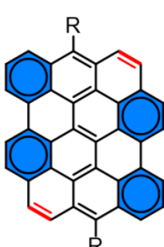

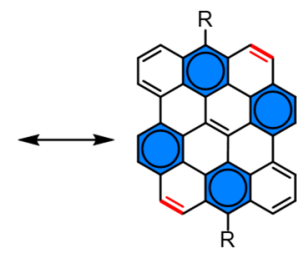

Figure 2. (a) X-ray crystallographic structure of DBOV-DMEP (measured at $193 \mathrm{~K}$ ): front view (left) and side view (right); (b) bond lengths from single-crystal analysis and NICS (0) values of each ring calculated at the GIAO-B3LYP/6-31G(d,p) level of theory using the Gaussian 09 simulation package; and (c) three resonance structures of the DBOV core with four Clar's aromatic sextet benzene rings indicated with circles and blue background.

essentially uniform in rings $A, D$, and $F$, while obvious alternations within a range of $0.05-0.30 \AA$ occur in rings $\mathrm{B}, \mathrm{C}$, and $\mathrm{E}$ (Figure $2 \mathrm{~b}$ ). In addition, the DBOV-DMEP molecules are staggered and exhibit a herringbone $\pi$-stacking motif in a layered structure. In the crystal, the face-to-face distance between two DBOV cores is $3.13 \AA$, which is shorter than the interlayer distance of graphite $(3.35 \AA)$. Close $\mathrm{CH}-\pi$ contact with a distance of $3.25 \AA$ is also observed (see Figure S5). Such close packing and strong intermolecular $\pi-\pi$ interactions are key features of high-performance, acene-based organic fieldeffect transistors (OFETs), ${ }^{49,50}$ which suggests the potential of DBOV-DMEP for applications in electronic devices.

The local aromaticity of individual rings was evaluated by means of nucleus-independent chemical shift (NICS) values calculated at the GIAO-B3LYP/6-31G(d,p) level of theory using the Gaussian 09 simulation package (see Supporting Information for details). ${ }^{51}$ Rings $\mathrm{A}, \mathrm{D}$, and $\mathrm{F}$ showed negative NICS values of $-7.41,-11.31$, and -9.35 , respectively, accounting for the experimentally observed chemical shifts of up to $9.6 \mathrm{ppm}$ for protons on these three rings. On the other hand, rings $\mathrm{B}, \mathrm{C}$, and $\mathrm{E}$ exhibited larger values of $-2.06,-4.05$, and 4.46, reflecting decreased aromaticity and antiaromaticity. Clar formulas could be drawn having benzene rings localized on these rings with negative NICS values (Figure 2c). Three of the Clar structures containing four benzene sextets are shown in Figure 2c. All structures possess two isolated double bonds in ring $\mathrm{B}$, which cannot be integrated into any benzene rings, explaining the observed upfield shifts of their proton signals $\left(\mathrm{H}_{3}\right.$ and $\left.\mathrm{H}_{4}\right)$ (Figure $1 \mathrm{a}$ ).

Photophysical and Electrochemical Properties. To understand the relationship between the peripheral substituents and the electronic structure of the DBOV core, their photophysical properties in solution were first investigated. Figure 3 illustrates the UV-vis absorption and fluorescence spectra of DBOV derivatives measured in toluene. These solutions showed well-resolved absorption bands between 450

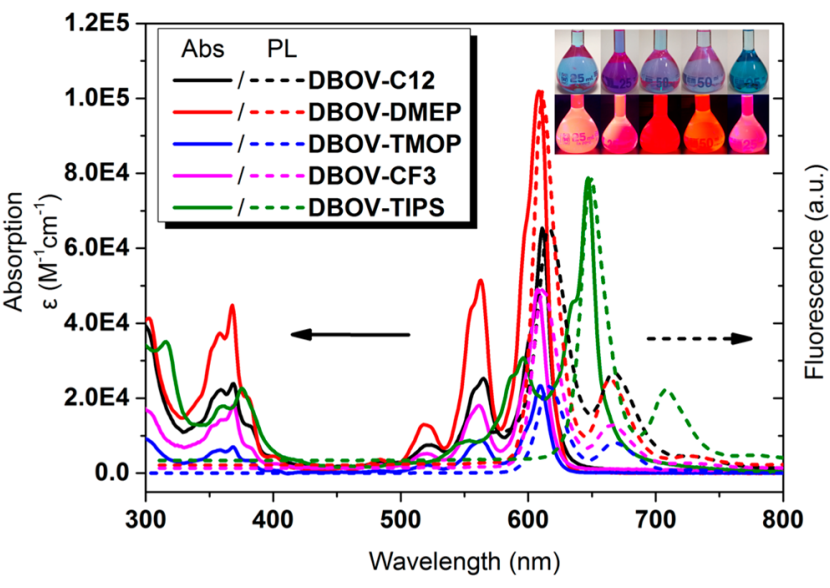

Figure 3. UV-vis absorption and fluorescence spectra of the DBOV derivatives measured in toluene solution at room temperature with a concentration of $10^{-5} \mathrm{M}$ (inset shows photographs of these solutions (top row) and fluorescence under UV light (bottom row); from left to right: DBOV-C12, DBOV-DMEP, DBOV-TMOP, DBOV-CF3, and DBOV-TIPS).

and $700 \mathrm{~nm}$ with maximum absorption peaks at $611 \mathrm{~nm}$ (DBOV-C12, molar extinction coefficient $\varepsilon=6.54 \times 10^{4} \mathrm{M}^{-1}$ $\mathrm{cm}^{-1}$ ), $608 \mathrm{~nm}$ (DBOV-DMEP, $\varepsilon=1.02 \times 10^{5} \mathrm{M}^{-1} \mathrm{~cm}^{-1}$ ), $609 \mathrm{~nm}$ (DBOV-TMOP, $\varepsilon=2.33 \times 10^{4} \mathrm{M}^{-1} \mathrm{~cm}^{-1}$ ), $607 \mathrm{~nm}$ (DBOV-TMOP, $\varepsilon=4.90 \times 10^{4} \mathrm{M}^{-1} \mathrm{~cm}^{-1}$ ), and $647 \mathrm{~nm}$ (DBOV-TIPS, $\varepsilon=7.88 \times 10^{4} \mathrm{M}^{-1} \mathrm{~cm}^{-1}$ ), which can be attributed mainly to the HOMO-LUMO transitions based on time-dependent density functional theory (TD-DFT) calculations (see Figure S7 and Table S2). At shorter wavelengths, vibronic replica involving $\mathrm{C}-\mathrm{C}$-stretching vibrations appear. ${ }^{28}$ The aryl substituents have a negligible influence on the UVvis absorption of the $\mathrm{DBOV}$ core due to the large dihedral angles and thus limited $\pi$-conjugation. In contrast, the maximum absorption peak of DBOV-TIPS is red-shifted by approximately $40 \mathrm{~nm}$, indicating efficient $\pi$-conjugation between the DBOV core and ethynyl groups. The absorption spectra showed no change after storing the solutions under air for 1 month, indicating the high stability of the DBOV core, which is an essential requirement for the applications of these materials in electronic and optical devices.

All derivatives showed strong fluorescence with maximum emission peaks located at $617 \mathrm{~nm}$ (DBOV-C12), $611 \mathrm{~nm}$ (DBOV-DMEP), $617 \mathrm{~nm}$ (DBOV-TTMOP), $611 \mathrm{~nm}$ (DBOV-CF3), and $650 \mathrm{~nm}$ (DBOV-TIPS). The small Stokes shifts of only 3-8 $\mathrm{nm}$ indicate a rigid structure of the core and small structural changes between the ground states and excited states. The fluorescence quantum yields (see Table 2) were determined against a Nile blue A perchlorate standard by measuring dilute solutions $(A<0.05)$ with decreasing concentrations. DBOV with alkyl or aryl groups exhibit high fluorescence quantum yields of $0.79-0.89$, while the comparatively low value of 0.67 observed for DBOV-TIPS is most likely due to the presence of the silylethynyl groups. The latter increase the vibronic coupling and enhance intersystem crossing. ${ }^{52}$ The optical gap of each derivative was estimated from the wavelength at which its absorption and fluorescence spectra cross each other, as listed in Table 2.

DFT calculations were performed to understand the effects of the substituents on the orbital energies. As shown in Table 2 and Figure S6, DBOV-CF3 possesses HOMO and LUMO levels at -4.72 and $-2.62 \mathrm{eV}$, respectively, which are lower 
Table 2. Optical and Electrochemical Properties of DBOV Derivatives

\begin{tabular}{|c|c|c|c|c|c|c|c|c|c|}
\hline compound & $\begin{array}{l}\lambda_{\max } \\
(\mathrm{nm})\end{array}$ & $\begin{array}{c}\lambda_{\mathrm{em}} \\
(\mathrm{nm})\end{array}$ & $\begin{array}{l}\lambda_{\text {edge }} \\
(\mathrm{nm})\end{array}$ & $\Phi$ & $\begin{array}{c}E_{\mathrm{g}}(\text { opt }) \\
(\mathrm{eV})^{a}\end{array}$ & $\begin{array}{c}E_{\mathrm{g}}(\mathrm{cal}) \\
(\mathrm{eV})^{b}\end{array}$ & $\begin{array}{l}\mathrm{HOMO}(\mathrm{cal}) \\
(\mathrm{eV})^{b}\end{array}$ & $\begin{array}{l}\text { LUMO (cal) } \\
\qquad(\mathrm{eV})^{b}\end{array}$ & $\begin{array}{c}\mathrm{HOMO}(\mathrm{CV}) \\
(\mathrm{eV})^{c}\end{array}$ \\
\hline DBOV-C12 & 611 & 617 & 621 & 0.79 & 2.02 & 2.10 & -4.46 & -2.37 & -4.72 \\
\hline DBOV-DMEP & 608 & 611 & 621 & 0.85 & 2.03 & 2.09 & -4.52 & -2.43 & -4.80 \\
\hline DBOV-TMOP & 609 & 617 & 623 & 0.86 & 2.02 & 2.10 & -4.47 & -2.37 & -4.57 \\
\hline DBOV-CF3 & 607 & 611 & 622 & 0.89 & 2.03 & 2.10 & -4.72 & -2.62 & -4.73 \\
\hline DBOV-TIPS & 647 & 650 & 661 & 0.67 & 1.91 & 1.90 & -4.58 & -2.68 & -4.71 \\
\hline
\end{tabular}

${ }^{a}$ Optical gaps were estimated based on the wavelength at which the normalized absorption and fluorescence spectra cross each other. ${ }^{b} \mathrm{DFT}$ calculations were performed at the B3LYP/6-31G $(\mathrm{d}, \mathrm{p})$ level of theory with the Gaussian 09 calculation package. ${ }^{{ }^{c}}$ The HOMO energy levels were calculated by using the onset of the first oxidation potential of $\mathrm{CV}$ calibrated with $\mathrm{Fc} / \mathrm{Fc}^{+}$.

than those of DBOV-C12, DBOV-DMEP, and DBOVTMOP, which are at approximately -4.5 and $-2.4 \mathrm{eV}$, respectively. DBOV-TIPS was calculated to have the lowest LUMO among the five DBOV derivatives, with an energy of $-2.68 \mathrm{eV}$, leading to a smaller HOMO-LUMO gap of $1.90 \mathrm{eV}$ compared to the gaps of approximately $2.1 \mathrm{eV}$ of the other cases. This trend observed for the calculated HOMO-LUMO gaps agrees well with that of the experimental optical gaps. The electrochemical properties of these DBOV derivatives were investigated by cyclic voltammetry $(\mathrm{CV})$ in dry dichloromethane solutions at room temperature. In the test window of the $\mathrm{CV}$, reversible oxidation waves were observed (Figure S8). The HOMO energy levels were calculated from the onset potential of the first oxidation wave using the following equation: $\mathrm{HOMO}=-\left(4.8+E_{\mathrm{ox}}{ }^{\text {onset }}\right)$, where the potentials were calibrated with $\mathrm{Fc} / \mathrm{Fc}^{+}$. The electrochemical HOMO levels approximately agreed with the calculated values (Table 2).

Single-Molecule Spectroscopy of DBOV-DMEP. Singlemolecule spectroscopy at room and low temperatures $(4.5 \mathrm{~K})$ was conducted with DBOV-DMEP embedded at very low concentrations in thin Zeonex polymer films. Although aggregation is unlikely under these conditions $\left(c \sim 10^{-10} \mathrm{M}\right.$ in solution), we chose this derivative because the 2,6dimethylphenyl groups would hinder the aggregation of the molecules. Benefiting from the large absorption cross-section and high fluorescence quantum yield of DBOV-DMEP, the single-molecule experiments provided deep insight into its photophysics, revealing many parameters previously unknown for DBOV.

Figure 4a displays the fluorescence emission spectra of two single DBOV-DMEP molecules (in Zeonex) measured at 296 and $4.5 \mathrm{~K}$. While the room-temperature spectrum closely resembles the bulk solution spectrum in toluene (see Figure 3 ), the spectrum at $4.5 \mathrm{~K}$ is characterized by a series of narrow vibronic transitions indicating weak linear electron-phonon coupling in the Zeonex host. The low-temperature spectrum clearly reveals that the broad bands observed at room temperature are composed of many vibronic transitions that are not resolved as a result of thermal broadening. The frequencies and relative intensities of the strongest vibrational transitions to the $S_{0}$ ground state determined from the lowtemperature emission spectrum (Figure $4 \mathrm{a}$, inset) are given in Table S3. As expected for a rigid planar $\mathrm{PAH}$, the purely electronic zero-phonon line (ZPL) has by far the highest intensity reflected in the large Franck-Condon factor of 0.36 . Since a normal-mode analysis is not currently available, we cannot assign the nature of the different vibrational modes. Qualitatively, literature data of other $\mathrm{PAHs}^{53,54}$ suggest that frequencies in the range of $100 \mathrm{~cm}^{-1}$ to $650 \mathrm{~cm}^{-1}$ belong to a)

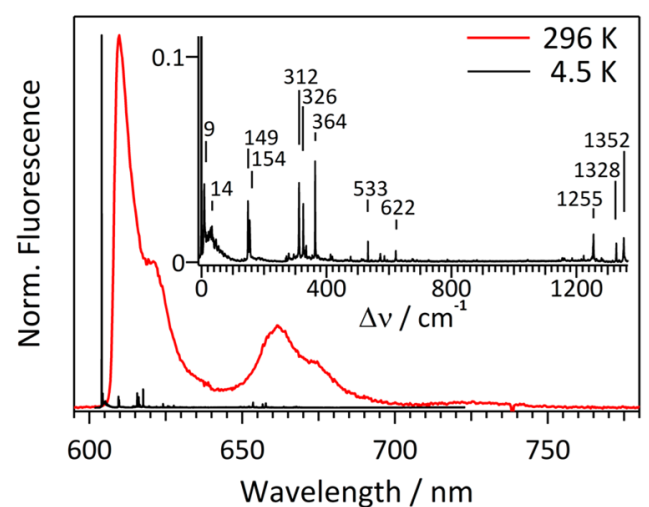

b)

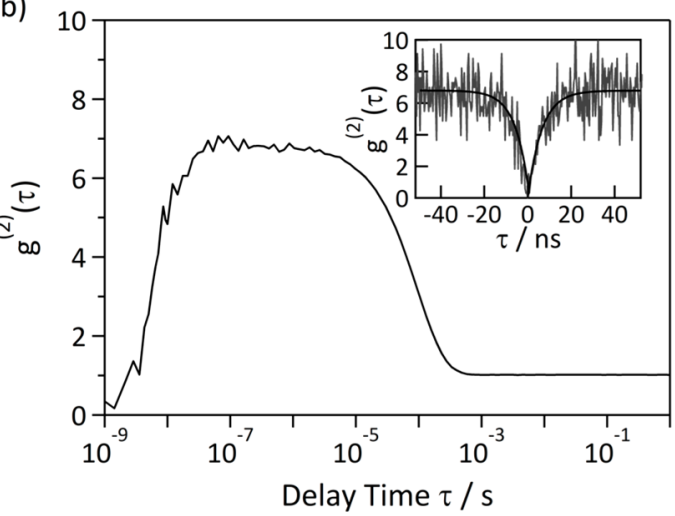

Figure 4. (a) Fluorescence spectra of two single DBOV-DMEP molecules embedded in a Zeonex film at $296 \mathrm{~K}\left(\right.$ red; $\left.\lambda_{\text {exc }}=561 \mathrm{~nm}\right)$ and $4.5 \mathrm{~K}$ (black; $\lambda_{\text {exc }}=565 \mathrm{~nm}$ ). The inset shows a magnified version of the high-resolution spectrum at $4.5 \mathrm{~K}$ with the vibronic transitions on an energy scale relative to the $[0,0]$-transition. (b) Fluorescence intensity autocorrelation function $g^{(2)}(\tau)$ of a single DBOV-DMEP molecule over 9 orders of magnitude in time $\left(I_{\text {exc }}=4 \mathrm{~kW} / \mathrm{cm}^{2}\right)$. The photon antibunching at short times is displayed in the inset on a linear time axis (also for negative delay times) together with a monoexponential fit to the data $\left(T=296 \mathrm{~K} ; \lambda_{\text {exc }}=561 \mathrm{~nm}\right)$.

totally symmetric modes involving movements of larger parts of the aromatic skeleton. In particular, we tentatively assume that the modes at $\sim 150 \mathrm{~cm}^{-1}$ are related to a long-axis breathing vibration of the whole molecule.

Since the temporal sequence of photons emitted by a single molecule constitutes a hallmark of its internal photophysical transitions, fluorescence correlation spectroscopy is an ideal tool to measure the rates of these transitions for immobilized single molecules. ${ }^{55-57}$ The fluorescence intensity autocorrelation function $g^{(2)}(\tau)$ of a single DBOV-DMEP molecule at room temperature covering 9 orders of magnitude in time is portrayed in Figure $4 \mathrm{~b} . g^{(2)}(\tau)$ displays the typical features 
expected in the photon statistics of a single organic dye molecule. At short times, the correlation function approaches zero, indicating single photon emission or photon antibunching, $^{58}$ while at longer times, the decay of the correlation function reveals photon bunching ${ }^{55}$ caused by the sequence of bright and dark periods as a result of transitions between the singlet and triplet manifolds.

To analyze the room-temperature correlation data, we treat the DBOV-DMEP molecule as an effective three-level system (Figure 5) and follow the pertinent procedures described in

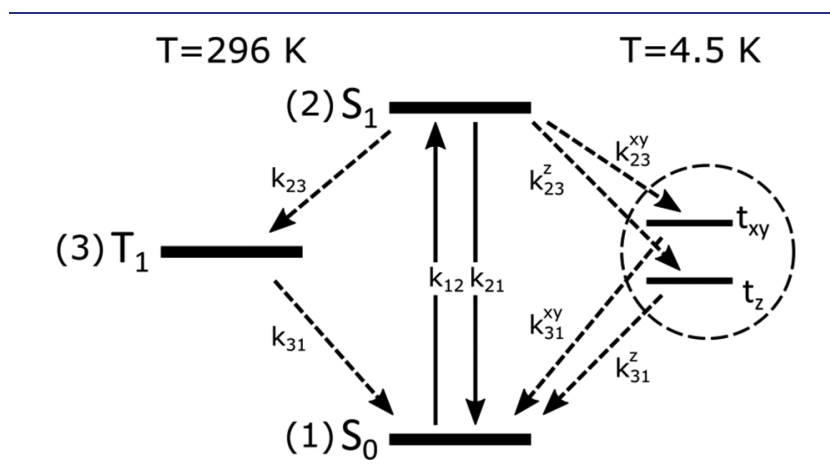

Figure 5. Simplified energy level scheme to highlight the relevant photophysical transitions in DBOV-DMEP. At $4.5 \mathrm{~K}$, the zero-field splitting of the triplet is taken into account. The $t_{x y}-t_{z}$-splitting is not to scale.

the literature. ${ }^{55,57,59}$ Since we excite the molecule nonresonantly into a higher energy vibronic level of $S_{1}$ at elevated temperature, we need not consider any coherences but can describe the transitions between levels by a system of rate equations. First, we want to focus on the behavior of the correlation function at short times. In the inset of Figure $4 b$, this part is displayed on an enlarged scale also for negative delay times. Without taking into account background contributions, ${ }^{55}$ which diminish the antibunching contrast, for the given intensity, $g^{(2)}(\tau)$ still approaches zero, i.e., $g^{(2)}(\tau=$ $0) \approx 0$. This fact clearly demonstrates that even at room temperature, single DBOV-DMEP molecules appear to be high-contrast single photon emitters. When increasing the excitation intensity, the contrast grows, and the rise of the correlation function becomes steeper, as clearly seen in Figure S9, where $g^{(2)}(\tau)$ is given for different intensities. The rise of $g^{(2)}(\tau)$ at short times has been approximated by the following expression (see SI):

$$
g^{(2)}(\tau)=A\left(1-e^{-\lambda_{\mathrm{a}}|\tau|}\right)+B
$$

$\lambda_{\mathrm{a}} \approx k_{21}+k_{12}$ and $k_{21}$ and $k_{12}$ denote the fluorescence decay and excitation rates, respectively. From the intensity dependence of $\lambda_{\mathrm{a}}\left(\lambda_{\mathrm{a}}\left(I_{\mathrm{exc}} \rightarrow 0\right) \approx k_{21}\right)$, the fluorescence decay rate can be obtained. ${ }^{57}$ The average $k_{21}$ value of four DBOV-DMEP molecules is given in Table 3 .

In the next step, the photon bunching part $(\mu \mathrm{s}-\mathrm{ms})$ of the room-temperature correlation data was analyzed to determine the ISC rate $k_{23}$ and the triplet decay rate $k_{31} \cdot g^{(2)}(\tau)$ data at long times were fitted for different excitation intensities by the following expression:

$$
g^{(2)}(\tau) \cong C e^{-\lambda_{\mathrm{b}} \tau}+1
$$

where $C$ is the contrast and $\lambda_{\mathrm{b}}$ is the decay parameter of the correlation function. From the intensity dependence of $C$ and $\lambda_{\mathrm{b}}$, the rates $k_{23}$ and $k_{31}$ were determined by globally fitting the
Table 3. Transition Rates of Single DBOV-DMEP and

\begin{tabular}{|c|c|c|c|c|c|}
\hline$T(\mathrm{~K})$ & $k_{21}\left(\mathrm{~s}^{-1}\right)$ & $k_{23}\left(\mathrm{~s}^{-1}\right)$ & $\begin{array}{l}k_{23}^{x y}\left(\mathrm{~s}^{-1}\right) \\
k_{23}^{z}\left(\mathrm{~s}^{-1}\right)\end{array}$ & $k_{31}\left(\mathrm{~s}^{-1}\right)$ & $\begin{array}{l}k_{31}^{x y}\left(\mathrm{~s}^{-1}\right) \\
k_{31}^{z}\left(\mathrm{~s}^{-1}\right)\end{array}$ \\
\hline \multicolumn{6}{|c|}{ DBOV-DMEP in Zeonex } \\
\hline 296 & $1.5 \times 10^{8}$ & $2.2 \times 10^{5}$ & - & $2.2 \times 10^{3}$ & - \\
\hline 4.5 & - & - & $\begin{array}{l}2.0 \times 10^{4} \\
1.6 \times 10^{3}\end{array}$ & - & $\begin{array}{l}2.0 \times 10^{3} \\
4.4 \times 10^{2}\end{array}$ \\
\hline \multicolumn{6}{|c|}{ Terrylene in $p$-Terphenyl } \\
\hline $300^{57}$ & - & $9.5 \times 10^{5}$ & & $6.9 \times 10^{3}$ & \\
\hline $1.4^{60}$ & - & - & $\begin{array}{l}2.0 \times 10^{3} \\
4.0 \times 10^{2}\end{array}$ & & $\begin{array}{l}1.9 \times 10^{3} \\
8.0 \times 10^{1}\end{array}$ \\
\hline
\end{tabular}
Terrylene Molecules ${ }^{a}$

${ }^{a} k_{21}$ : fluorescence decay rate; $k_{23}$ : intersystem crossing rate; $k_{31}$ : triplet decay rate. DBOV-DMEP: $296 \mathrm{~K}$ (average of 4 molecules); $4.5 \mathrm{~K}$ (average of 13 molecules).

appropriate equations to the data (see SI). The average values of both rates for the same four molecules for which the fluorescence decay rate $k_{21}$ was determined are given in Table 3.

The experiments described above were conducted under an argon atmosphere. Preliminary experiments under air have indicated that both $k_{23}$ and $k_{31}$ increase, indicating a quenching of $S_{1}$ and $T_{1}$ by oxygen. ${ }^{61}$ Moreover, photobleaching becomes much more efficient, most likely caused by a reaction of the electron-rich $\mathrm{PAH}$ with singlet oxygen, which is formed via quenching of the triplet state $\mathrm{T}_{1}{ }^{62}$

$g^{(2)}(\tau)$ at long times was also measured for single DBOVDMEP molecules at low temperature $(4.5 \mathrm{~K})$. Under these conditions, the correlation function could be well approximated by a biexponential decay (Figure 6a), as has been found in single-molecule studies of other PAHs, such as terrylene ${ }^{63}$ and perylene. ${ }^{64}$ At low temperatures, the distributed kinetics of the triplet sublevels originating from the zero-field splitting of the triplet state emerge because spin-lattice relaxation is largely suppressed. Given the low symmetry of the molecule $\left(C_{2 h}\right)$, the splitting should result in three sublevels. As shown here by X-ray analysis of DBOV-DMEP crystals, the molecules adopt a nearly planar core. For planar PAHs-with the molecular plane as the sufficient symmetry element-it typically has been found that the out-of-plane sublevel $\left(t_{z}\right)$ is characterized by smaller rates in and out of the triplet state compared to the two in-plane levels. ${ }^{63-68}$ The kinetics of the latter two are often similar and difficult to distinguish in the correlation decay. In the remainder of the text, we will treat the two in-plane levels as a single level $t_{x y}$, keeping in mind that the actual relation between the molecular $x$ and $y$ axes and the magnetic axes is not known for DBOV-DMEP. Accordingly, the biexponential decay of the correlation function reflects the population and depopulation kinetics of the triplet sublevels $t_{x y}$ and $t_{z}$. The pertinent rates again have been determined from the intensity dependence of the corresponding contrasts $C_{\mathrm{i}}$ and decay parameters $\lambda_{\mathrm{i}}$ of the correlation function, following a procedure from the literature. ${ }^{64}$ This analysis is different from the treatment of the room-temperature data as outlined in the SI. Moreover, to fit the intensity dependences of $C_{\mathrm{i}}$ and $\lambda_{\mathrm{i}}$ adequately, we had to introduce an additional intensity dependence of the triplet decay rates leading to apparent rates $K_{31}^{\mathrm{i}}=k_{31}^{\mathrm{i}}+\alpha_{\mathrm{i}} \cdot I_{\text {exc }}$. We assume that the additional term results from triplet-triplet absorption, as has been proposed in other studies. 57,69 This process shows up in the lowtemperature experiments because we had to use high laser 
a)

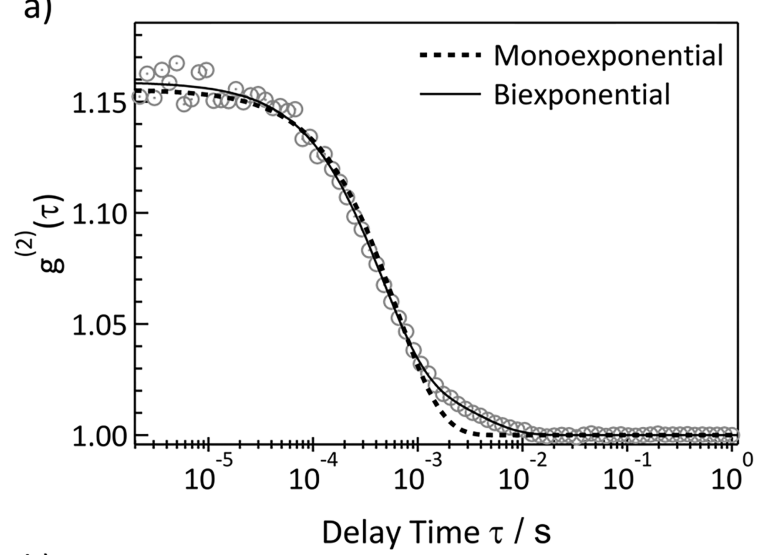

b)

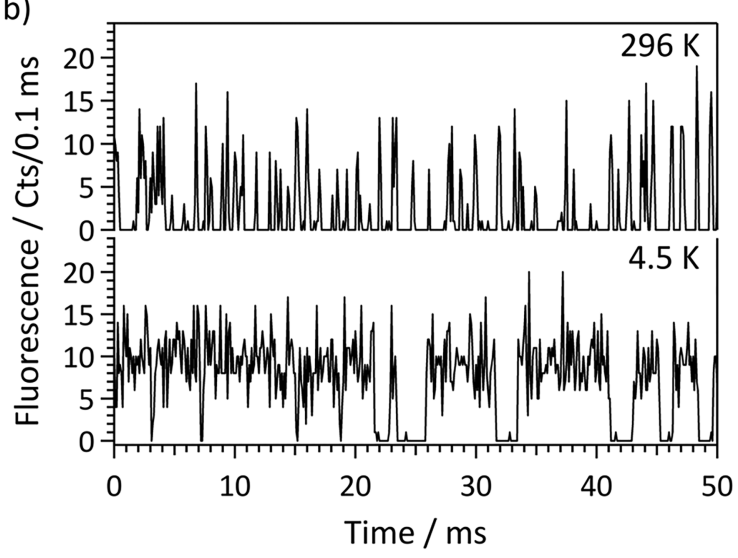

Figure 6. (a) Fluorescence intensity autocorrelation function $g^{(2)}(\tau)$ of a single DBOV-DMEP molecule at long times $\left(I_{\mathrm{exc}}=42 \mathrm{~kW} / \mathrm{cm}^{2}\right.$, $T=4.5 \mathrm{~K}$ ). Monoexponential (dashed line) and biexponential (drawn line) fits to the data are also shown. (b) Fluorescence counts as a function of time for two DBOV-DMEP molecules at the given temperatures. The different appearances of the two traces reflect the temperature dependence of the ISC rate $k_{23}$.

intensities due to the very weak absorption of DBOV-DMEP at the excitation wavelength.

In Table 3, the average values of the various population and depopulation rates $\left(k_{23}^{x y}, k_{23}^{z}, k_{31}^{x y}, k_{31}^{z}\right)$ at $4.5 \mathrm{~K}$ are listed. The ISC rates $k_{23}^{x y}$ and $k_{23}^{z}$ differ by a factor of 13 , and the triplet decay rates $k_{31}^{x y}$ and $k_{31}^{z}$ differ by a factor of 5 , supporting the distinction in terms of in-plane and out-of-plane sublevels.

To compare the room and low temperature rates, we refer to the values of the $t_{x y}$ level for the latter, since those will dominate the kinetics at elevated temperatures. While $k_{31}$ basically did not change between room and low temperature, $k_{23}$ decreased on average by more than an order of magnitude at $4.5 \mathrm{~K}$. (At both temperatures, the molecules were studied under noble gas atmospheres.) This remarkable change in the ISC rate is nicely visualized in Figure $6 \mathrm{~b}$, where the fluorescence intensity as a function of time is displayed for two DBOV-DMEP molecules at the two temperatures. For these particular molecules, which were studied at comparable excitation rates to minimize the effects caused by the intensity dependence of $k_{23}$, the ISC rates differed by a factor of 30. At room temperature, only very short bright intervals are visible because the molecule quickly crosses from the singlet state $S_{1}$ into the triplet state $T_{1}$, and no emission occurs. In contrast, much longer bright periods are observed at low temperature, where the probability for ISC is substantially reduced. In other words, the number of single photons emitted before the molecule crosses into the triplet state is significantly enhanced. Obviously, for an ideal single photon emitter, ISC should be absent. We note that the rates $k_{31}$ can be directly extracted from such traces, since the lengths of the dark intervals are directly related to the $T_{1}$ lifetime. $^{60}$

The temperature dependence of $S_{1}-T_{1}$ ISC has been studied for several PAHs, particularly linear polyacenes such as naphthalene and anthracene. ${ }^{66,70-72}$ In general, ISC will be temperature dependent if higher vibrational levels $v$ of $S_{1}\left(S_{1, v}\right)$ have a different mechanism for the nonradiative transition to $\mathrm{T}_{1}$ than the vibrationally relaxed $\mathrm{S}_{1,0}$ level. In most cases, the temperature dependence of the ISC rate has been expressed as a thermally activated process, by which a higher triplet state $T_{n}$ $(n>1)$ is populated at elevated temperatures. Typically, such studies have been based on the measurement of the changes in the fluorescence quantum yield or intensity with temperature. We note that such an approach is feasible only when the ISC rate can compete with the radiative decay rate of $S_{1}$, which is obviously not the case for DBOV-DMEP. For anthracene crystals, it was found that in the range of $140-430 \mathrm{~K}$, the temperature dependence of ISC follows a thermally activated process with an activation energy of $800 \mathrm{~cm}^{-1}{ }^{70}$ Studies of brominated anthracenes in 3-methylpentane over a comparable temperature range gave similar activation energies. ${ }^{72}$ For pentacene $/ p$-terphenyl mixed crystals, the temperature dependence of the pentacene ISC rate between 4.2 and $140 \mathrm{~K}$ was attributed to a vibronically induced process due to a lowfrequency $\left(\sim 30 \mathrm{~cm}^{-1}\right)$ out-of-plane vibrational mode of pentacene. $^{71}$ Although the details of the coupling mechanism between $S_{1}$ and $T_{1}$ can be quite involved and diverse,${ }^{66}$ owing to the varying contributions of, e.g., direct and vibronically induced spin-orbit coupling for different species, the common conclusion for the few systems studied appears to be that the thermal population of higher vibrational levels of $S_{1}$ lowers the energy gap to higher triplet states $T_{n}(n>1)$, thereby increasing the ISC rate.

To date, most of the single-molecule studies in which the ISC and triplet decay rates have been determined for a particular dye molecule were performed either at low temperature or at room temperature by employing the correlation method..$^{55,73-75}$ (A compilation of mainly lowtemperature data can be found in the literature. ${ }^{64}$ ) To our knowledge, only the mixed crystalline system terrylene $/ p$ terphenyl has been investigated at room temperature and at 1.4 $\mathrm{K}$, but these experiments have been performed in different laboratories. ${ }^{57,63}$ The $k_{23}$ and $k_{31}$ values for single terrylene molecules at different temperatures are given in Table 3 . In this case, the $k_{23}$ value decreases by more than 2 orders of magnitude at low temperature, while the triplet lifetime changes only slightly. Unfortunately, for this system, a comparison is also complicated by the fact that the $p$-terphenyl crystal undergoes an order/disorder phase-transition at $\sim 190$ $\mathrm{K}$, and the relation between molecules from the four lowtemperature sites $^{63}$ and the molecules in the crystal under ambient conditions is not clear. Nevertheless, the same trend as observed for the ovalene is noticed for terrylene.

Since the measurements of DBOV-DMEP were performed at only two temperatures, the functional form of the temperature dependence of $k_{23}$ could not be established. As discussed above, the increased ISC rate at room temperature could be caused by the thermal population of vibrational modes of $S_{1}$. In addition to an activated process, a static 
distortion of the molecule could lead to increased ISC rates because in a nonplanar geometry of the ovalene core, spinorbit coupling might be enhanced due to $\sigma-\pi$ interactions. $^{74,76,77}$ However, DBOV-DMEP is a relatively rigid structure and was observed to be planar in the crystal. In addition, it is not obvious why a change in temperature should affect the degree of static distortion in the rigid Zeonex host. Although we cannot completely rule out a distortion of the molecules as the origin of the increased ISC rate at room temperature, we feel that the description by an activated process, which has been reported for several PAHs, is more appealing. To shed more light on this issue, it would be most helpful to measure the ISC rate over the whole temperature range from 4.5 to $296 \mathrm{~K}$ for a given single molecule. Such an experiment, however, is challenging due to the enhanced photobleaching probability at elevated temperatures.

\section{CONCLUSION AND OUTLOOK}

In summary, we have achieved efficient syntheses of a series of DBOV derivatives with different meso-substituents by using a photochemical cyclodehydroiodination reaction (PCDHI) as the key step. The number of synthesis steps could be reduced from 12 to 7, and the total yield was improved from approximately $2 \%$ up to approximately $40 \%$, which enabled scalable syntheses of different DBOV derivatives. 2,6Dimethylphenyl groups as the substituents allowed for the unambiguous single-crystal X-ray analysis, revealing the planar structure of the DBOV core. Relatively uniform bond lengths observed in rings $\mathrm{A}, \mathrm{D}$, and $\mathrm{F}$ indicated the localization of aromatic sextets in these benzene rings, in accordance with the results derived from ${ }^{1} \mathrm{H}$ NMR experiments and NICS calculations. UV-vis absorption measurements showed that the aliphatic and aromatic substituents had minor effects on the electronic properties of the DBOV core, while a redshift of the optical absorption by approximately $40 \mathrm{~nm}$ could be achieved through the introduction of the TIPS-ethynyl group. Strong red fluorescence was observed for all DBOVs in toluene solutions, with a high relative fluorescence quantum yield of up to 0.89 , showing the promise of these molecules in applications as light-emitting materials.

Our comprehensive single-molecule spectroscopy study of DBOV-DMEP at room and low temperatures has provided novel insights into the photophysics of this ovalene derivative. By analyzing the fluorescence correlation function at long times, the triplet kinetics could be assessed. While the triplet decay rate basically did not change between room and low temperature, the ISC rate decreased by more than an order of magnitude at low temperature. On the basis of a comparison to literature data, we concluded that the increase in the ISC rate at room temperature results from the thermal population of higher vibrational levels of $S_{1}$, which decreases the energy gap to higher-lying triplet states $T_{n}(n>1)$ and accelerates the ISC. The observation of high-contrast photon antibunching combined with a high fluorescence yield and photostability as well as the presence of intense ZPLs at low temperature qualify DBOV-DMEP as a promising single quantum emitter, which is ideally suited for high-resolution, frequency-resolved single-molecule spectroscopy. While lowering the temperature typically is considered advantageous mainly because of the appearance of sharp ZPLs and the increased photostability of single molecules, our results imply that low temperatures can also improve the intrinsic dynamics of a single photon emitter.
The current results thus provide not only a reliable synthetic route to obtain large amounts of various DBOV derivatives but also deeper insights into its structural, optoelectronic, and photophysical properties. These findings lay the foundation for the further development of DBOV as a promising luminescent nanographene material. Studies on further derivatizations of DBOV and the reactivity of its peripheral positions as well as applications in optoelectronic devices are ongoing. With respect to the photophysics of nanographenes, it would be most valuable to disclose how the actual rate constants depend on the chemical structure and energy level scheme of a particular compound. Along these lines, it will be interesting to study photophysical changes occurring from bisanthene to the parent ovalene, whereby a structure with armchair edges develops into a structure with zigzag edges only.

\section{EXPERIMENTAL SECTION}

Lightly doped polymer films for the single molecule measurements were prepared by spin-coating a toluene solution of DBOV-DMEP and the polymer (Nippon Zeon K.K., Zeonex 330R, $20 \mathrm{~g} / \mathrm{L}$ ) at 4000 $\mathrm{rpm}$ onto glass substrates. The concentration of the dye in the solution was $\sim 10^{-10} \mathrm{M}$. For the room temperature measurements borosilicate glass substrates were used, while at $4.5 \mathrm{~K}$ fused silica was employed. All substrates were cleaned following standard procedures.

Single molecule measurements at room temperature were done using a home-built confocal setup. Briefly, the output of a fiber coupled CW solid state laser (Toptica iChrome CLE, $561 \mathrm{~nm}$ ) was collimated and focused onto the sample plane by an oil-immersion objective (Zeiss, Plan-Apochromat 100×/1.4), after having passed a $565 \mathrm{~nm}$ band-pass filter and being reflected off a beam splitter (80T:20R). Residual excitation light in the detection path was blocked by a $594 \mathrm{~nm}$ long-pass filter before the fluorescence light was divided by a beam splitter (50T:50R). One-half was split again (50T:50R) and detected by two APDs in a Hanbury-Brown Twiss configuration to circumvent detector dead times. The other half was dispersed by a spectrograph (Acton Spectra Pro 300i, resolution: 25 $\mathrm{cm}^{-1}$ ) and detected with a CCD camera (Andor, Newton EM-CCD).

Measurements at $4.5 \mathrm{~K}$ were performed with a customized variable temperature confocal microscope (attocube, CFM1). The excitation light from a CW solid-state laser (Coherent Sapphire, $568 \mathrm{~nm}$ ) first was guided through a single-mode fiber for spatial filtering. After collimation, the beam passed a $565 \mathrm{~nm}$ band-pass filter and was reflected by a beam splitter (80T:20R) into the back aperture of a low-temperature objective (attocube, LT-APO/VISIR, NA = 0.82), mounted in the cryostat. The fluorescence, collected by the same objective, was cleaned up by a $594 \mathrm{~nm}$ long-pass filter after passing the exit window of the cryostat and guided through a multimode fiber (NA: 0.1). The fluorescence light emerging from the fiber was collimated and directed to a beam splitter (50T:50R). The transmitted light was focused onto an APD, while the reflected light was focused onto the entrance slit of a spectrograph (Andor, Spectra Pro HRS-750-B1-R, resolution: $\sim 1 \mathrm{~cm}^{-1}$ in high-resolution mode) equipped with a CCD camera (Andor, Newton EM-CCD).

In both setups the photon counts of the APDs were fed into a TCSPC module (PicoQuant, PicoHarp 300, time-tagged timeresolved (TTTR) mode). Fluorescence intensity autocorrelation functions were calculated from the time delays between the photon arrival times.

\section{ASSOCIATED CONTENT}

\section{Supporting Information}

The Supporting Information is available free of charge on the ACS Publications website at DOI: 10.1021/jacs.9b08320.

Further experimental details, single-crystal data for $\mathbf{6}$ and DBOV-DMEP, NMR, MS, IR, UV-vis absorption and 
fluorescence spectra, cyclic voltammetry, calculation details, single molecule data and analysis (PDF)

Crystal data for 6 (CIF)

Crystal data for DBOV-DMEP (CIF)

\section{AUTHOR INFORMATION}

\section{Corresponding Authors}

*muellen@mpip-mainz.mpg.de

*narita@mpip-mainz.mpg.de

*thomas.basche@uni-mainz.de

ORCID

Qiang Chen: 0000-0001-5612-1504

Klaus Müllen: 0000-0001-6630-8786

Akimitsu Narita: 0000-0002-3625-522X

\section{Author Contributions}

${ }^{\#}$ Q.C. and S.T. contributed equally to this work.

\section{Notes}

The authors declare the following competing financial interest(s): Qiang Chen, Klaus Müllen, and Akimitsu Narita are listed as inventors on patent applications (application no. 18199451.8 - EPO and application no. 18199447.6 - EPO) related to the work presented in this manuscript. All other authors have nothing to disclose.

\section{ACKNOWLEDGMENTS}

This work was financially supported by Max Planck Society and the European Union's Horizon 2020 research and innovation programme under Marie-Curie ITN project "iSwitch" (GA No.642196).

\section{REFERENCES}

(1) Son, Y. W.; Cohen, M. L.; Louie, S. G. Half-metallic graphene nanoribbons. Nature 2006, 444 (7117), 347-9.

(2) Trauzettel, B.; Bulaev, D. V.; Loss, D.; Burkard, G. Spin qubits in graphene quantum dots. Nat. Phys. 2007, 3 (3), 192-196.

(3) Nakada, K.; Fujita, M.; Dresselhaus, G.; Dresselhaus, M. S. Edge state in graphene ribbons: Nanometer size effect and edge shape dependence. Phys. Rev. B: Condens. Matter Mater. Phys. 1996, 54 (24), 17954-17961.

(4) Son, Y. W.; Cohen, M. L.; Louie, S. G. Energy gaps in graphene nanoribbons. Phys. Rev. Lett. 2006, 97 (21), 216803.

(5) Hod, O.; Barone, V.; Scuseria, G. E. Half-metallic graphene nanodots: A comprehensive first-principles theoretical study. Phys. Rev. B: Condens. Matter Mater. Phys. 2008, DOI: 10.1103/ PhysRevB.77.035411.

(6) Barone, V.; Hod, O.; Scuseria, G. E. Electronic structure and stability of semiconducting graphene nanoribbons. Nano Lett. 2006, 6 (12), 2748-54.

(7) Döhnert, D.; Koutecky, J. Occupation numbers of natural orbitals as a criterion for biradical character. Different kinds of biradicals. J. Am. Chem. Soc. 1980, 102 (6), 1789-1796.

(8) Li, X.; Wang, X.; Zhang, L.; Lee, S.; Dai, H. Chemically derived, ultrasmooth graphene nanoribbon semiconductors. Science 2008, 319 (5867), 1229-32.

(9) Jeon, S.-C.; Kim, Y.-S.; Lee, D.-K. Fabrication of a Graphene Nanoribbon with Electron Beam Lithography Using a XR-1541/ PMMA Lift-Off Process. Trans. Electr. Electron. Mater. 2010, 11 (4), 190-193.

(10) Lemme, M. C.; Bell, D. C.; Williams, J. R.; Stern, L. A.; Baugher, B. W.; Jarillo-Herrero, P.; Marcus, C. M. Etching of graphene devices with a helium ion beam. ACS Nano 2009, 3 (9), 2674-6.

(11) Ruffieux, P.; Cai, J.; Plumb, N. C.; Patthey, L.; Prezzi, D.; Ferretti, A.; Molinari, E.; Feng, X.; Müllen, K.; Pignedoli, C. A.; Fasel,
R. Electronic structure of atomically precise graphene nanoribbons. ACS Nano 2012, 6 (8), 6930-5.

(12) Tapaszto, L.; Dobrik, G.; Lambin, P.; Biro, L. P. Tailoring the atomic structure of graphene nanoribbons by scanning tunnelling microscope lithography. Nat. Nanotechnol. 2008, 3 (7), 397-401.

(13) Bai, J.; Duan, X.; Huang, Y. Rational fabrication of graphene nanoribbons using a nanowire etch mask. Nano Lett. 2009, 9 (5), 2083-7.

(14) Kosynkin, D. V.; Higginbotham, A. L.; Sinitskii, A.; Lomeda, J. R.; Dimiev, A.; Price, B. K.; Tour, J. M. Longitudinal unzipping of carbon nanotubes to form graphene nanoribbons. Nature 2009, 458 (7240), 872-6.

(15) Jiao, L.; Zhang, L.; Wang, X.; Diankov, G.; Dai, H. Narrow graphene nanoribbons from carbon nanotubes. Nature 2009, 458 (7240), 877-80.

(16) Narita, A.; Wang, X. Y.; Feng, X.; Müllen, K. New advances in nanographene chemistry. Chem. Soc. Rev. 2015, 44 (18), 6616-43.

(17) Chen, L.; Hernandez, Y.; Feng, X.; Müllen, K. From nanographene and graphene nanoribbons to graphene sheets: chemical synthesis. Angew. Chem., Int. Ed. 2012, 51 (31), 7640-54.

(18) Wang, X.-Y.; Narita, A.; Müllen, K. Precision synthesis versus bulk-scale fabrication of graphenes. Nat. Rev. Chem. 2018, DOI: $10.1038 / \mathrm{s} 41570-017-0100$.

(19) Li, J.; Zhang, K.; Zhang, X.; Huang, K. W.; Chi, C.; Wu, J. meso-Substituted bisanthenes as soluble and stable near-infrared dyes. J. Org. Chem. 2010, 75 (3), 856-63.

(20) Ni, Y.; Gopalakrishna, T. Y.; Phan, H.; Herng, T. S.; Wu, S.; Han, Y.; Ding, J.; Wu, J. A Peri-tetracene Diradicaloid: Synthesis and Properties. Angew. Chem., Int. Ed. 2018, 57 (31), 9697-9701.

(21) Ajayakumar, M. R.; Fu, Y.; Ma, J.; Hennersdorf, F.; Komber, H.; Weigand, J. J.; Alfonsov, A.; Popov, A. A.; Berger, R.; Liu, J.; Müllen, K.; Feng, X. Toward Full Zigzag-Edged Nanographenes: periTetracene and Its Corresponding Circumanthracene. J. Am. Chem. Soc. 2018, 140 (20), 6240-6244.

(22) Konishi, A.; Hirao, Y.; Nakano, M.; Shimizu, A.; Botek, E.; Champagne, B.; Shiomi, D.; Sato, K.; Takui, T.; Matsumoto, K.; Kurata, H.; Kubo, T. Synthesis and characterization of teranthene: a singlet biradical polycyclic aromatic hydrocarbon having Kekule structures. J. Am. Chem. Soc. 2010, 132 (32), 11021-3.

(23) Konishi, A.; Hirao, Y.; Matsumoto, K.; Kurata, H.; Kishi, R.; Shigeta, Y.; Nakano, M.; Tokunaga, K.; Kamada, K.; Kubo, T. Synthesis and characterization of quarteranthene: elucidating the characteristics of the edge state of graphene nanoribbons at the molecular level. J. Am. Chem. Soc. 2013, 135 (4), 1430-7.

(24) Sun, Z.; Zeng, Z.; Wu, J. Benzenoid polycyclic hydrocarbons with an open-shell biradical ground state. Chem. - Asian J. 2013, 8 (12), 2894-904.

(25) Sun, Z.; Ye, Q.; Chi, C.; Wu, J. Low band gap polycyclic hydrocarbons: from closed-shell near infrared dyes and semiconductors to open-shell radicals. Chem. Soc. Rev. 2012, 41 (23), 7857-89.

(26) Liu, J.; Ravat, P.; Wagner, M.; Baumgarten, M.; Feng, X.; Müllen, K. Tetrabenzo[a,f,j,o]perylene: a polycyclic aromatic hydrocarbon with an open-shell singlet biradical ground state. Angew. Chem., Int. Ed. 2015, 54 (42), 12442-6.

(27) Arabei, S. M.; Pavich, T. A. Spectral-luminescent properties and photoinduced transformations of bisanthene and bisanthenequinone. J. Appl. Spectrosc. 2000, 67 (2), 236-244.

(28) Paterno, G. M.; Chen, Q.; Wang, X. Y.; Liu, J.; Motti, S. G.; Petrozza, A.; Feng, X.; Lanzani, G.; Müllen, K.; Narita, A.; Scotognella, F. Synthesis of Dibenzo[hi,st] ovalene and Its Amplified Spontaneous Emission in a Polystyrene Matrix. Angew. Chem., Int. Ed. 2017, 56 (24), 6753-6757.

(29) Coles, D. M.; Chen, Q.; Flatten, L. C.; Smith, J. M.; Müllen, K.; Narita, A.; Lidzey, D. G. Strong Exciton-Photon Coupling in a Nanographene Filled Microcavity. Nano Lett. 2017, 17 (9), 55215525.

(30) Stabel, A.; Herwig, P.; Müllen, K.; Rabe, J. P. Diodelike Current-Voltage Curves for a Single Molecule-Tunneling Spectros- 
copy with Submolecular Resolution of an Alkylated,peri-Condensed Hexabenzocoronene. Angew. Chem., Int. Ed. Engl. 1995, 34 (15), $1609-1611$

(31) Iyer, V. S.; Wehmeier, M.; Brand, J. D.; Keegstra, M. A.; Müllen, K. From Hexa-peri-hexabenzocoronene to "Superacenes". Angew. Chem., Int. Ed. Engl. 1997, 36 (15), 1604-1607.

(32) Watson, M. D.; Fechtenkötter, A.; Müllen, K. Big Is Beautiful-"Aromaticity" Revisited from the Viewpoint of Macromolecular and Supramolecular Benzene Chemistry. Chem. Rev. 2001, 101 (5), 1267-1300.

(33) King, B. T.; Kroulik, J.; Robertson, C. R.; Rempala, P.; Hilton, C. L.; Korinek, J. D.; Gortari, L. M. Controlling the Scholl reaction. J. Org. Chem. 2007, 72 (7), 2279-88.

(34) Liu, J.; Narita, A.; Osella, S.; Zhang, W.; Schollmeyer, D.; Beljonne, D.; Feng, X.; Müllen, K. Unexpected Scholl Reaction of 6,7,13,14-Tetraarylbenzo[k]tetraphene: Selective Formation of FiveMembered Rings in Polycyclic Aromatic Hydrocarbons. J. Am. Chem. Soc. 2016, 138 (8), 2602-8.

(35) Wadumethrige, S. H.; Rathore, R. A facile synthesis of elusive alkoxy-substituted hexa-peri-hexabenzocoronene. Org. Lett. 2008, 10 (22), 5139-42.

(36) Ormsby, J. L.; Black, T. D.; Hilton, C. L.; Bharat; King, B. T. Rearrangements in the Scholl oxidation: implications for molecular architectures. Tetrahedron 2008, 64 (50), 11370-11378.

(37) Graczyk, A.; Murphy, F. A.; Nolan, D.; Fernandez-Moreira, V.; Lundin, N. J.; Fitchett, C. M.; Draper, S. M. Terpyridine-fused polyaromatic hydrocarbons generated via cyclodehydrogenation and used as ligands in $\mathrm{Ru}(\mathrm{II})$ complexes. Dalton. Trans. 2012, 41 (25), $7746-54$.

(38) Yang, W.; Lucotti, A.; Tommasini, M.; Chalifoux, W. A. Bottom-Up Synthesis of Soluble and Narrow Graphene Nanoribbons Using Alkyne Benzannulations. J. Am. Chem. Soc. 2016, 138 (29), 9137-44.

(39) Yang, W.; Longhi, G.; Abbate, S.; Lucotti, A.; Tommasini, M.; Villani, C.; Catalano, V. J.; Lykhin, A. O.; Varganov, S. A.; Chalifoux, W. A. Chiral Peropyrene: Synthesis, Structure, and Properties. J. Am. Chem. Soc. 2017, 139 (37), 13102-13109.

(40) Li, C. W.; Wang, C. I.; Liao, H. Y.; Chaudhuri, R.; Liu, R. S. Synthesis of dibenzo[g,p]chrysenes from bis(biaryl)acetylenes via sequential ICl-induced cyclization and Mizoroki-Heck coupling. J. Org. Chem. 2007, 72 (24), 9203-7.

(41) Zhu, C.; Wang, D.; Wang, D.; Zhao, Y.; Sun, W. Y.; Shi, Z. Bottom-up Construction of pi-Extended Arenes by a PalladiumCatalyzed Annulative Dimerization of o-Iodobiaryl Compounds. Angew. Chem., Int. Ed. 2018, 57 (29), 8848-8853.

(42) Daigle, M.; Picard-Lafond, A.; Soligo, E.; Morin, J. F. Regioselective Synthesis of Nanographenes by Photochemical Cyclodehydrochlorination. Angew. Chem., Int. Ed. 2016, 55 (6), 2042-7.

(43) Mohamed, R. K.; Mondal, S.; Guerrera, J. V.; Eaton, T. M.; Albrecht-Schmitt, T. E.; Shatruk, M.; Alabugin, I. V. Alkynes as Linchpins for the Additive Annulation of Biphenyls: Convergent Construction of Functionalized Fused Helicenes. Angew. Chem., Int. Ed. 2016, 55 (39), 12054-8.

(44) Pun, S. H.; Chan, C. K.; Luo, J.; Liu, Z.; Miao, Q. A Dipleiadiene-Embedded Aromatic Saddle Consisting of 86 Carbon Atoms. Angew. Chem., Int. Ed. 2018, 57 (6), 1581-1586.

(45) Cheung, K. Y.; Chan, C. K.; Liu, Z.; Miao, Q. A Twisted Nanographene Consisting of 96 Carbon Atoms. Angew. Chem., Int. Ed. 2017, 56 (31), 9003-9007.

(46) Kawasumi, K.; Zhang, Q.; Segawa, Y.; Scott, L. T.; Itami, K. A grossly warped nanographene and the consequences of multiple oddmembered-ring defects. Nat. Chem. 2013, 5 (9), 739-44.

(47) Fukuyama, T.; Fujita, Y.; Miyoshi, H.; Ryu, I.; Kao, S. C.; Wu, Y. K. Electron transfer-induced reduction of organic halides with amines. Chem. Commun. (Cambridge, U. K.) 2018, 54 (44), 55825585.

(48) Liu, J.; Li, B. W.; Tan, Y. Z.; Giannakopoulos, A.; SanchezSanchez, C.; Beljonne, D.; Ruffieux, P.; Fasel, R.; Feng, X.; Müllen, K.
Toward cove-edged low band gap graphene nanoribbons. J. Am. Chem. Soc. 2015, 137 (18), 6097-103.

(49) Mattheus, C. C.; Dros, A. B.; Baas, J.; Meetsma, A.; Boer, J. L. d.; Palstra, T. T. M. Polymorphism in pentacene. Acta Crystallogr., Sect. C: Cryst. Struct. Commun. 2001, 57 (8), 939-941.

(50) Watanabe, M.; Chang, Y. J.; Liu, S. W.; Chao, T. H.; Goto, K.; Islam, M. M.; Yuan, C. H.; Tao, Y. T.; Shinmyozu, T.; Chow, T. J. The synthesis, crystal structure and charge-transport properties of hexacene. Nat. Chem. 2012, 4 (7), 574-8.

(51) Schleyer, P. V. R.; Maerker, C.; Dransfeld, A.; Jiao, H.; van Eikema Hommes, N. J. R. Nucleus-Independent Chemical Shifts: A Simple and Efficient Aromaticity Probe. J. Am. Chem. Soc. 1996, 118 (26), 6317-6318.

(52) Yamaji, M.; Maeda, H.; Nanai, Y.; Mizuno, K. Substitution effects of CC triple bonds on the fluorescent properties of perylenes studied by emission and transient absorption measurements. Chem. Phys. Lett. 2012, 536, 72-76.

(53) Kummer, S.; Kulzer, F.; Kettner, R.; Basché, T.; Tietz, C.; Glowatz, C.; Kryschi, C. Absorption, excitation, and emission spectroscopy of terrylene in p-terphenyl: Bulk measurements and single molecule studies. J. Chem. Phys. 1997, 107 (19), 7673-7684.

(54) D’Yachenko, G. G.; Petukhov, V. A.; Arabei, S. M.; Pavich, T. A. Analysis of the Electronic Vibrational Spectra of Bisanthene by Calculating Normal Modes. J. Appl. Spectrosc. 2003, 70 (2), 208-215.

(55) Bernard, J.; Fleury, L.; Talon, H.; Orrit, M. Photon bunching in the fluorescence from single molecules: A probe for intersystem crossing. J. Chem. Phys. 1993, 98 (2), 850-859.

(56) Weston, K. D.; Carson, P. J.; DeAro, J. A.; Buratto, S. K. Singlemolecule detection fluorescence of surface-bound species in vacuum. Chem. Phys. Lett. 1999, 308 (1-2), 58-64.

(57) Fleury, L.; Segura, J.; Zumofen, G.; Hecht, B.; Wild, U. P. Nonclassical photon statistics in single-molecule fluorescence at room temperature. Phys. Rev. Lett. 2000, 84 (6), 1148-51.

(58) Basché, T.; Moerner, W. E.; Orrit, M.; Talon, H. Photon antibunching in the fluorescence of a single dye molecule trapped in a solid. Phys. Rev. Lett. 1992, 69 (10), 1516-1519.

(59) Novotny, L.; Hecht, B. Principles of Nano-Optics; Cambridge University Press: Cambridge, 2012.

(60) Basché, T.; Kummer, S.; Bräuchle, C. Direct spectroscopic observation of quantum jumps of a single molecule. Nature 1995, 373 (6510), 132-134.

(61) Turro, N. J.; Ramamurthy, V.; Scaiano, J. C. Modern Molecular Photochemistry of Organic Molecules; University Science Books: Sausalito, 2010.

(62) Christ, T.; Kulzer, F.; Bordat, P.; Basché, T. Watching the Photo-Oxidation of a Single Aromatic Hydrocarbon Molecule. Angew. Chem., Int. Ed. 2001, 40 (22), 4192-4195.

(63) Kummer, S.; Basché, T.; Bräuchle, C. Terrylene in p-terphenyl: a novel single crystalline system for single molecule spectroscopy at low temperatures. Chem. Phys. Lett. 1994, 229 (3), 309-316.

(64) Verhart, N. R.; Navarro, P.; Faez, S.; Orrit, M. Intersystem crossing rates of single perylene molecules in ortho-dichlorobenzene. Phys. Chem. Chem. Phys. 2016, 18 (26), 17655-9.

(65) Henry, B. R.; Siebrand, W. Spin-Orbit Coupling in Aromatic Hydrocarbons. Analysis of Nonradiative Transitions between Singlet and Triplet States in Benzene and Naphthalene. J. Chem. Phys. 1971, 54 (3), 1072-1085.

(66) Lawetz, V.; Orlandi, G.; Siebrand, W. Theory of Intersystem Crossing in Aromatic Hydrocarbons. J. Chem. Phys. 1972, 56 (8), 4058-4072.

(67) Metz, F.; Friedrich, S.; Hohlneicher, G. What is the leading mechanism for the nonradiative decay of the lowest triplet state of aromatic hydrocarbons? Chem. Phys. Lett. 1972, 16 (2), 353-358.

(68) Bräuchle, C. Symmetry distortions and pseudo-Jahn-Teller effect of peri- and cata-hexabenzocoronene in their triplet states as obsevered by ODMR techniques. Chem. Phys. 1982, 67 (1), 97-109.

(69) Banasiewicz, M.; Morawski, O.; Wia̧cek, D.; Kozankiewicz, B. Triplet population and depopulation rates of single terrylene 
molecules in p-terphenyl crystal. Chem. Phys. Lett. 2005, 414 (4-6), 374-377.

(70) Adolph, J.; Williams, D. F. Temperature Dependence of Singlet-Triplet Intersystem Crossing in Anthracene Crystals. J. Chem. Phys. 1967, 46 (11), 4248-4251.

(71) Kryschi, C.; Wagner, B.; Gorgas, W.; Schmid, D. Vibronically induced intersystem crossing in pentacene in p-terphenyl and benzoic acid crystals. J. Lumin. 1992, 53 (1-6), 468-472.

(72) Hamanoue, K.; Nakayama, T.; Ikenaga, K.; Ibuki, K. Temperature effect on the formation of the lowest excited triplet states of 9-bromoanthracene and 9,10-dibromoanthracene. J. Photochem. Photobiol., A 1993, 74 (2-3), 147-152.

(73) Boiron, A. M.; Lounis, B.; Orrit, M. Single molecules of dibenzanthanthrene in n-hexadecane. J. Chem. Phys. 1996, 105 (10), 3969-3974.

(74) Lang, E.; Hildner, R.; Engelke, H.; Osswald, P.; Wurthner, F.; Kohler, J. Comparison of the photophysical parameters for three perylene bisimide derivatives by single-molecule spectroscopy. ChemPhysChem 2007, 8 (10), 1487-96.

(75) Blom, H.; Chmyrov, A.; Hassler, K.; Davis, L. M.; Widengren, J. Triplet-state investigations of fluorescent dyes at dielectric interfaces using total internal reflection fluorescence correlation spectroscopy. $J$. Phys. Chem. A 2009, 113 (19), 5554-66.

(76) Clarke, R. H.; Frank, H. A. Triplet state radiationless transitions in polycyclic hydrocarbons. J. Chem. Phys. 1976, 65 (1), 39-47.

(77) Wu, Y.; Zhen, Y.; Ma, Y.; Zheng, R.; Wang, Z.; Fu, H. Exceptional Intersystem Crossing in Di(perylene bisimide)s: A Structural Platform toward Photosensitizers for Singlet Oxygen Generation. J. Phys. Chem. Lett. 2010, 1 (17), 2499-2502. 